\title{
Caracterização morfológica de piperáceas nativas conservadas em casa de vegetação
}

\author{
Morphological characterization of native piperacea conserved in vegetation house \\ Caracterización morfológica de piperacea nativas conservada en casa de vegetación
}

Recebido: 02/05/2021 | Revisado: 10/05/2021 | Aceito: 13/05/2021 | Publicado: 31/05/2021

Danielle Santana Rito

ORCID: https://orcid.org/0000-0002-3432-4332

Universidade Federal Rural da Amazônia, Brasil

E-mail: daniellesrito@gmail.com

Eduardo Filipe Torres Vieira

ORCID: https://orcid.org/0000-0002-7696-7838

Universidade Federal Rural da Amazônia, Brasil

E-mail: Eduardo_filipe16@yahoo.com.br

Ilmarina Campos de Menezes

ORCID: https://orcid.org/0000-0003-4795-3151

Embrapa Amazônia Oriental, Brasil

E-mail: ilmarina.menezes@embrapa.br

Osmar Alves Lameira

ORCID: https://orcid.org/0000-0001-8370-8562

Embrapa Amazônia Oriental, Brasil

E-mail: osmar.lameira@embrapa.br

Marli Costa Poltronieri

ORCID: https://orcid.org/0000-0002-3871-854X Embrapa Amazônia Oriental, Brasil

E-mail: marli.poltronieri@embrapa.br

Oriel Filgueira de Lemos

ORCID: https://orcid.org/0000-0002-7331-1024 Embrapa Amazônia Oriental, Brasil

E-mail: oriel.lemos@embrapa.br

Simone de Miranda Rodrigues

ORCID: https://orcid.org/0000-0001-5717-785X Embrapa Amazônia Oriental, Brasil

E-mail: simone.rodrigues@embrapa.br

\begin{abstract}
Resumo
A família Piperácea possui diversas espécies de interesse econômico, medicinal, ornamental e ambiental. A Embrapa Amazônia Oriental possui uma coleção de piperáceas nativas para garantir a diversidade dessas espécies e o uso em programas de melhoramento genético, dentre outras finalidades. Esse trabalho teve como objetivo descrever as características morfológicas de quatro espécies de piperáceas conservadas em casa de vegetação: Piper marginatum Jacq., Piper montealegreanum Yunck, Piper peltatum L. e Piper tuberculatum Jacq. Foi mensurado o comprimento e largura de folhas, pedúnculo, pecíolo, espigas, entrenós, e altura das plantas. Também foi avaliado o hábito de crescimento, filotaxia das folhas, formato de folhas e caule, presença de lenticelas e ataque de pragas e doenças nos espécimes. Documentou-se a coloração de folhas, caules e espigas usando a carta de Munsell. Os dados consideraram a média das medidas aferidas de quatro espécimes, por espécie, para cada órgão avaliado e comparado a informações da literatura. Os resultados demonstraram que ocorreram diferenças nas características morfológicas das plantas quando cultivadas em ambientes controlados.
\end{abstract}

Palavras-chave: Casa de vegetação; Piper L., Piperáceas nativas; Plantas medicinais.

\begin{abstract}
The Piperácea family has several species of economic, medicinal, ornamental and environmental interest. Embrapa Amazônia Oriental has a collection of native piperaceae to ensure the diversity of these species and their use in breeding programs, among other finalities. This work aimed to describe the morphological characteristics of four species of piperacea preserved in a greenhouse: Piper marginatum Jacq., Piper montealegreanum Yunck, Piper peltatum L. and Piper tuberculatum Jacq. The length and width of leaves, peduncle, petiole, reproductive structures, internodes, and plant height were measured. Growth habit, phyllotaxis of leaves, shape of leaves and stem, presence of lenticels and attack of pests and diseases on specimens were also evaluated. The coloring of leaves, stems and ears was documented using the Munsell chart. The data considered the average of the measurements taken from four specimens, per species, for each evaluated organ and compared to information from the literature. The results showed that there were differences in the morphological characteristics of the plants when grown in controlled environments.
\end{abstract}

Keywords: Greenhouse; Medicinal plants; Native piperaceae; Piper L. 


\begin{abstract}
Resumen:
La familia Piperácea tiene varias especies de interés económico, medicinal, ornamental y ambiental. Embrapa Amazônia Oriental cuenta con una colección de piperaceae nativas para garantizar la diversidad de estas especies y su uso en programas de mejoramiento genético, entre otros fines. Este trabajo tuvo como objetivo describir las características morfológicas de cuatro especies de piperacea conservadas en invernadero: Piper marginatum Jacq., Piper montealegreanum Yunck, Piper peltatum L. y Piper tuberculatum Jacq. Se midieron el largo y ancho de las hojas, pedúnculo, pecíolo, mazorcas, entrenudos y altura de la planta. También se evaluó el hábito de crecimiento, filotaxis de hojas, forma de hojas y tallo, presencia de lenticelas y ataque de plagas y enfermedades en los ejemplares. La coloración de hojas, tallos y mazorcas se documentó mediante la tabla de Munsell. Los datos consideraron el promedio de las medidas tomadas de cuatro especímenes, por especie, para cada órgano evaluado y su compararon con información de la literatura. Los resultados mostraron que hubo diferencias en las características morfológicas de las plantas cuando se cultivaron en ambientes controlados.
\end{abstract}

Palabras clave: Invernadero; Piper L.; Piperaceae nativa; Plantas medicinales.

\title{
1. Introdução
}

A família Piperaceae tem grande importância em todo o território no qual é distribuída, sendo considerada por Yuncker (1958) uma família possível de ser encontrada nas porções tropicais dos continentes Americano, Asiático e Africano. Estima-se que a família possua aproximadamente 3600 espécies (Smith et al., 2008) distribuídas nessas porções tropicais. Apresenta ampla importância econômica, medicinal, ornamental e ambiental, justificando seu interesse científico. No Brasil, a família Piperaceae é distribuída em cinco gêneros, sendo que os gêneros Piper L. e Peperomia Ruiz \& Pav. abrangem o maior número de espécies (Yuncker, 1972).

Na região amazônica, espécies nativas como Piper peltatum L., $P$. marginatum Jacq., $P$. tuberculatum Jacq. e $P$. montealegreanum Yunck são conhecidas como plantas medicinais podendo ser usadas contra insetos, picadas de cobras, e como analgésico ou anti-inflamatório (D'Angelo et al., 1997; De Araújo Júnior et al., 1999; Pinto et al., 2012). Um exemplo é a espécie Piper marginatum Jacq., popularmente conhecida como pimenta-do-mato ou malvaísco, que tem suas raízes utilizadas no tratamento de picada de insetos, contra doenças hepáticas e tem potencial larvicida contra Aedes aegypti e Leishmania amazonensis (De Oliveira Chaves \& De Oliveira Santos, 2002; Autran et al., 2009; Pereira et al., 2020; Macêdo et al., 2020). Também pode ser usada na agricultura, no combate ao fungo Cladosporium cladosporioides, mostrando eficiência, principalmente no período de pós-colheita (Macêdo et al., 2020). Atualmente, há uma grande preocupação em relação às perdas de recursos genéticos da biodiversidade, em especial a conservação da diversidade genética do Brasil, que conta com cerca de $24 \%$ da flora mundial. Essa biodiversidade costuma ser conservada principalmente in situ ou ex situ.

A conservação in situ representa a proteção das espécies em seus habitats naturais permitindo a continuidade evolutiva e o sustentáculo da vida silvestre. Em contrapartida, a conservação ex situ consiste na manutenção da diversidade genética fora de seu habitat natural para diversos fins que vão desde a preservação genética e proteção da diversidade até a conservação in vitro que contribui com o melhoramento genético, entre outras formas de uso (Brasil, 2012). Já a conservação on farm está diretamente ligada à conservação in situ, no entanto, é utilizada por pequenos agricultores em suas fazendas com o cultivo de espécies silvestres ou crioulas, como são conhecidas. O cultivo é feito, normalmente, em pequenas comunidades que são consideradas o centro de origem da variedade agrícola tradicional alvo da conservação (Clement et al., 2007; Brasil, 2012).

A Embrapa Amazônia Oriental, como estratégia de conservação, mantém em casa de vegetação uma coleção de piperáceas nativas visando preservar a diversidade de espécies da família Piperaceae, o enriquecimento, a multiplicação, o intercâmbio e a caracterização dos materiais, com possibilidade de uso em projetos de melhoramento genético e medicinal, dentre outras finalidades. Atualmente conta com 18 espécies nativas, as quais se adicionaram à coleção de cultivares de pimenta-do-reino (Piper nigrum L.) pertencente à mesma instituição. Esse trabalho objetivou a descrição morfológica de quatro espécies de piperáceas conservadas em casa de vegetação, por estarem identificadas botanicamente e possuírem 
escassas informações na literatura.

\section{Metodologia}

A obtenção dos dados ocorreu durante o primeiro semestre de 2020 na Embrapa Amazônia Oriental, em Belém (PA), que conta com uma coleção de espécies de Piperaceaes nativas oriundas de coletas realizadas em anos anteriores na Serra dos Carajás (PA). A coleção é mantida em sacos para mudas, medindo $20 \times 25 \mathrm{~cm}$, e estão conservadas em casa de vegetação e apresentam condições de temperatura que giram em torno de 25 a 35 graus celsius. Diariamente são irrigadas e a cada seis meses são submetidos à adubação foliar usando o Complex 151 (nitrogênio 5\%, fósforo 11\%, potássio 16\%, cálcio 2\%, magnésio $2,0 \%$, enxofre $5 \%$, boro $1,5 \%$, cobre $0,5 \%$, ferro $0,1 \%$, manganês $0,5 \%$, molibdênio: $0,2 \%$, zinco $4 \%$ )

A caracterização morfológica de espécies tem grande relevância por ser um método simples e preciso em relação a sua menor interferência perante os efeitos do ambiente quando comparados a características agronômicas ou fenotípicas (Vieira et al., 2007; Lameira et al., 2020), por conta disso, foram realizadas análises qualitativas, com avaliação das características morfológicas dos espécimes para quatro espécies, com identificação botânica confirmada previamente, como segue: P. marginatum Jacq., P. montealegreanum Yunk., P. peltatum L. e P. tuberculatum Jacq, sendo uma metodologia rotineiramente realizada para estudos de conservação, caracterização e melhoramento genético (Rodrigues, Hirt \& Flores, 2012; Pereira et al., 2018; Mendoza et al., 2018; Silva et al., 2020; Silva et al., 2021). Foi realizada a mensuração dos tamanhos com relação às medidas do comprimento e largura de folhas, comprimento de pedúnculo e pecíolo, tamanho das espigas, quando presentes, comprimento de entrenós e altura das plantas, utilizando paquímetro, régua e trena. Ilustrações baseadas nos espécimes tipo (feitas por Eduardo Filipe Torres Vieira) e escaneadas, destacam características morfológicas para cada espécie, são apresentadas.

Para as espécies, avaliou-se o hábito de crescimento, filotaxia das folhas, formato de folhas e caule, presença de lenticelas e ataque de pragas e doenças nos espécimes utilizados. Também, foi observada a coloração de folhas, caule e espiga com auxílio do sistema de cores de Munsell (Munsell, 1905) a qual foi elaborada uma listagem (Tabela 1) para auxiliar na descrição das colorações. Os dados consideraram a média de aferição das medidas obtidas de quatro espécimes por espécie para cada órgão avaliado. 
Tabela 1 - Descrição das cores obtidas através do sistema de colorações de Munsell.

\begin{tabular}{|c|c|c|}
\hline Código da coloração & Nome da coloração & Tradução \\
\hline 3GY 3304 & Lt yellow green & Verde-amarelo claro \\
\hline 3GY 3305 & Bright yellow green & Verde-amarelo brilhante \\
\hline 3GY 3307 & Dp yellow green & Verde-amarelo profundo \\
\hline 3GY 3309 & Dk green & Verde Escuro \\
\hline 3YR 1308 & Dp brown & Marrom profundo \\
\hline 3YR 1309 & Brown & Marrom \\
\hline 3YR 1318 & Dk grayish brown & Marrom-acinzentado escuro \\
\hline 5GY 3504 & Bright yellow green & Verde-amarelo brilhante \\
\hline 5GY 3508 & Dk yellow green & Verde-amarelo escuro \\
\hline 5GY 3512 & Strong yellow green & Verde-amarelo intenso \\
\hline 5GY 3513 & Dull yellow green & Verde-amarelo opaco \\
\hline 7GY 3707 & Dk green & Verde escuro \\
\hline 7GY 3710 & Strong yellowish green & Verde- amarelado intenso \\
\hline 7GY 3711 & Dp yellowish green & Verde- amarelado profundo \\
\hline 9YR 1908 & Dp yellowish orange & Laranja-amarelado profundo \\
\hline
\end{tabular}

Fonte: Autores.

\section{Resultados e Discussão}

As avaliações das plantas indicaram que a espécie $P$. marginatum Jacq. exibiu hábito de crescimento ereto, folhas em formato oval cordiforme, caule em formato cilíndrico, filotaxia alterna e com presença de lenticelas. Essa espécie apresentou $91,7 \mathrm{~cm}$ de altura, folhas maduras medindo $13,9 \mathrm{~cm}$ de comprimento por 14,29 cm de largura, e entrenós e pecíolos com 12,16 $\mathrm{cm}$ e 5,52 cm, respectivamente, espigas com 6,88 cm de comprimento e pedúnculo medindo 0,58 cm. A carta de Munsell indicou folhas maduras com coloração verde escuro (7GY 3707), as folhas jovens apresentaram coloração verde-amarelo escuro (5GY 3508), o caule maduro de cor verde-amarelo opaco (5GY 3513), enquanto que o caule jovem de cor verdeamarelo intenso (5GY 3512). Foram identificados espécimes suscetíveis a patógenos e a pragas desfolhadoras.

A literatura cita que Piper montealegreanum Yunk. tem componentes com ação antibacteriana e hemolítica (Pinto et al., 2012; Rocha et al., 2020). Essa espécie apresentou hábito de crescimento arbustivo, folhas em formato oval lanceolada, caule em formato cilíndrico, filotaxia alterna e com ausência de lenticelas. A espécie analisada apresentou em média 36,5 cm de altura, folhas medindo $10,8 \mathrm{~cm}$ de comprimento por 4,42 cm de largura, entrenós e pecíolos com $3,7 \mathrm{~cm}$ e $0,8 \mathrm{~cm}$ de comprimento, respectivamente, espigas com $3,95 \mathrm{~cm}$ e pedúnculos com $0,35 \mathrm{~cm}$ de comprimento. Identificou-se a presença de pólen em algumas espigas, espigas mais jovens, que apresentaram média de comprimento de 1,9 cm. A carta de Munsell mostrou a coloração verde-amarelo escuro (5GY 3508) para as folhas maduras, a coloração verde-amarelo brilhante (5GY 3504) para as folhas jovens, a coloração marrom- acinzentado escuro (3YR 1318) para o caule maduro, a coloração marrom (3YR 1309) para o caule jovem, a coloração verde-amarelo claro (3GY 3304) para o broto e a coloração verde-amarelo brilhante (3GY 3305) para as espigas maduras. Não foi constatado o surgimento de doenças ou ataque de pragas nas plantas avaliadas.

A espécie Piper peltatum L. é comumente utilizada como diurético, possui compostos anti-inflamatórios, podendo apresentar efeito bactericida (De Feo, 1991; Pascoli et al., 2018). No presente trabalho apresentou hábito de crescimento arbustivo, folhas em formato cordada por apresentar lobos arredondados na base das folhas formando um coração (Judd et al., 2009), caule em formato cilíndrico, filotaxia alterna verticilada e ausência de lenticelas. A espécie analisada apresentou 71,5 $\mathrm{cm}$ de altura, folhas medindo $27,21 \mathrm{~cm}$ de comprimento por 29,15 cm de largura, entrenó e pecíolo medindo 5,67 cm e 21,9 
$\mathrm{cm}$, respectivamente, espigas medindo 6,2 $\mathrm{cm}$ de comprimento, e pedúnculo apresentando $0,8 \mathrm{~cm}$. A carta de Munsell mostrou a coloração verde-amarelo escuro (5GY 3508) para as folhas maduras, a coloração verde- amarelado profundo (7GY 3711) para o caule maduro e a coloração verde-amarelado intenso (7GY 3710) para o caule jovem. Os espécimes analisados apresentaram ataque por pragas desfolhadoras, mas não apresentavam doenças.

Piper tuberculatum Jacq. é popularmente conhecida como pimenta-de-macaco ou pimenta-longa, e é utilizada contra problemas respiratórios, como asma e bronquite, e contra diarreia (Dos Santos Sales et al., 2017). Também apresenta resistência ao Fusarium solani, que é o principal fungo que acomete a pimenta-do-reino (Pereira et al., 2019). A espécie apresentou hábito de crescimento arbustivo, folhas em formato oval lanceolada, caule em formato cilíndrico, filotaxia alterna verticilada e presença de lenticelas. Os espécimes analisados apresentaram $54,75 \mathrm{~cm}$ de altura, folhas medindo $13,72 \mathrm{~cm}$ de comprimento por $5,41 \mathrm{~cm}$ de largura, entrenó e pecíolo com $5,85 \mathrm{~cm}$ e $0,85 \mathrm{~cm}$, respectivamente, espigas com $3,2 \mathrm{~cm}$ de comprimento, e pedúnculo com $0,7 \mathrm{~cm}$. As folhas maduras apresentaram coloração verde Escuro (3GY 3309), enquanto que as folhas jovens apresentaram coloração verde-amarelo profundo (3GY 3307), a coloração laranja-amarelado profundo (9YR 1908) para caule maduro e a coloração marrom profundo (3YR 1308) para caule jovem.

Na coleção de espécies de Piperaceaes nativas da Embrapa é possível notar algumas diferenças em relação às mesmas espécies quando alocadas em diferentes locais ou idade da planta analisada, como altura da planta, tamanho de estruturas e presença de espigas. Essas variações podem ser observadas em relação à espécie P. marginatum Jacq. identificadas por Melo (2013), que relatou que o espécime examinado apresentou três metros de altura e as folhas medindo em média $12,75 \mathrm{~cm}$ de comprimento por $10 \mathrm{~cm}$ de largura. O tamanho das folhas encontrado neste trabalho com o apresentado por Melo (2013) foi semelhante, no entanto, a altura apresentou uma discrepância de aproximadamente dois metros, sendo esperado devido à incidência luminosa e as condições naturais favoráveis ao desenvolvimento da espécie. O elevado potencial contra as larvas do Aedes aegypti apresentado pela espécie ocorre pela presença de sesquiterpenos no óleo essencial extraído de suas folhas, sendo que tal ação larvicida é maior do que na espécie Piper nigrum L. que é a espécie de maior importância econômica da família (Costa et al., 2009).

A Figura 1 apresenta o desenho dos principais órgãos vegetais das quatro espécies de piperáceas consideradas neste estudo, sendo possível visualizar diferenças anatômicas e morfológicas entre as espécies consideradas. 
Figura 1 - Piperáceas nativas da Amazônia, evidenciando principalmente as diferenças morfológicas entre as folhas das espécies avaliadas neste estudo. Piper montealegreanum Yunk. (A e E); Piper marginatum Jacq. (B e F); Piper tuberculatum Jacq. (C e G); Piper peltatum L. (D e H). O tamanho das barras escuras de 2 e $4 \mathrm{~cm}$ ao lado das figuras representam uma estimativa do tamanho real dos tecidos, órgãos vegetais e altura da planta.

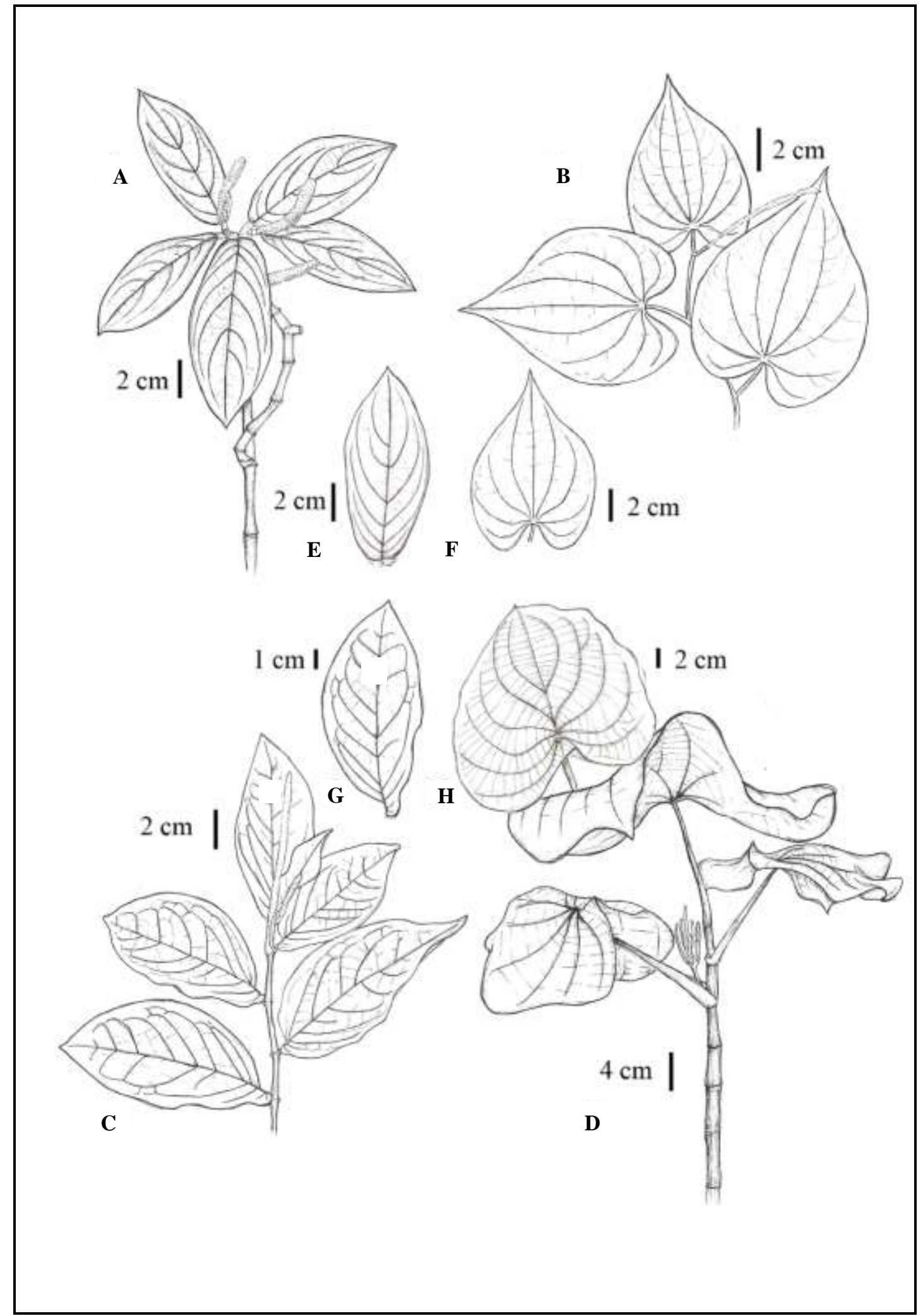

Fonte: Autores.

A espécie P. montealegreanum Yunk expressou em testes laboratoriais que seus dois principais flavonóides, 
denominados PMT1 e PMT2, apresentaram acentuados efeitos antinociceptivos e anti-inflamatórios e por conta disso demonstram elevado potencial farmacêutico para a criação de novas opções de analgésicos e anti-inflamatórios no mercado (De Queiroz et al., 2014). A espécie foi catalogada pela primeira vez no ano de 1908 e ainda sim continua com poucos estudos morfológicos acerca da mesma.

Segundo a literatura, a espécie Piper peltatum L. é descrita por Guimarães \& Giordano (2004) como um arbusto com até $2 \mathrm{~m}$ de altura, diferente dos espécimes contidos na casa de vegetação. No entanto, os tamanhos de folha e pecíolo encontrados nos espécimes mantidos em casa de vegetação, foram aproximados aos da literatura e as características morfológicas descritas foram condizentes com o relatado para a espécie. Perigo et al. (2016) encontrou como principal componente químico no óleo essencial extraído dessa espécie os sesquiterpenóides de carbono que apresenta alta atividade antibacteriana e que inibe o crescimento do número de bactérias.

Segundo Guimarães e Giordano (2004), a espécie Piper tuberculatum Jacq. é uma planta arbustiva que mede entre 2 a 2,5 m de altura em condições naturais. Entretanto, quanto conservadas em casa de vegetação apresentaram 1/5 desse tamanho, em consequência do ambiente ao qual os espécimes estão alocados. Sarnaglia Junior, Bermudez e Guimarães (2013) afirmam que a espécie em campo apresenta pecíolo puberulento e os nós apresentando tubérculos, características essas que ocorrem também nos espécimes mantidos em casa de vegetação. Sobreira, Sobreira, Hernandez e Souza (2017) afirmam que se pode utilizar o óleo essencial de $P$. tuberculatum como inseticida na agricultura orgânica por conta do seu vasto potencial fitoquímico, no entanto não há estudos na região amazônica que constatem seu efeito em uma espécie em uma espécie específica.

$\mathrm{Na}$ Tabela 2 podem-se observar de forma sucinta, as aferições das características morfológicas das espécies que estão conservadas no banco ativo de germoplasma da Embrapa Amazônia Oriental em condições de casa de vegetação e o nome popular das mesmas.

Tabela 2 - Medidas das características morfológicas das espécies (cm).

\begin{tabular}{ccccccc}
\hline Espécie & $\begin{array}{c}\text { Altura da } \\
\text { planta }\end{array}$ & Folha (C x L) & Entrenó & Pecíolo & Espiga & Pedúnculo \\
\hline P. marginatum Jacq. & 91,7 & $13,9 \times 14,29$ & 12,16 & 5,52 & 6,88 & 0,58 \\
P. montealegreanum Yunk. & 36,5 & $10,8 \times 4,42$ & 3,7 & 0,8 & 3,95 & 0,35 \\
P. peltatum L. & 71,5 & $27,21 \times 29,15$ & 5,67 & 21,9 & 6,2 & 0,8 \\
P. tuberculatum Jacq. & 54,75 & $13,72 \times 5,41$ & 5,85 & 0,85 & 3,2 & 0,7 \\
\hline
\end{tabular}

Fonte: Autores.

As plantas de piperácea mantidas em casa de vegetação passam por poda anual, podendo ser uma das causas da variação relativa às alturas dos espécimes, entretanto, os valores dos apêndices dos mesmos foram proporcionais aos descritos na literatura para as espécies consideradas, sendo considerada interessante e eficiente essa forma de conservação para essas espécies, visto ocuparem menos espaço que em condições naturais, serem facilmente controláveis e não necessitarem de pessoal de campo e manejo específico para sua manutenção e multiplicação, como ocorre no campo.

\section{Conclusão}

Ocorrem diferenças nas características morfológicas das espécies de piperáceas apresentadas neste trabalho, em decorrência da conservação em ambiente controlado, entretanto, mostra-se como uma alternativa eficiente para a manutenção. Trabalhos futuros comparando a composição química dos principais metabólitos secundários produzidos por essas espécies tornam-se interessantes para verificar as principais diferenças metabólicas encontradas em cultivos controlados, considerando- 
se a importância medicinal dessas espécies. Ainda, pesquisas complementares focadas na descrição de descritores morfoagronômicos, estudos reprodutivos e de produtividade associados à fenologia das espécies, conservação in vitro e multiplicação em larga escala possibilitarão a domesticação dessas espécies de piperáceas e facilitarão a expansão do uso em indústrias químicas, farmacêuticas e, também, universidades, direcionando programas de melhoramentos de espécies relacionadas de interesse econômico, assim como, desenvolvendo produtos como bioinsumos e/ou biopesticidas úteis para a agricultura.

\section{Agradecimentos}

À Embrapa Amazônia Oriental pelo apoio financeiro via projeto No. 11.15.02.002.08.00 (PC8: Bancos de Germoplasma de Plantas Medicinais, Aromáticas, Inseticidas, Corantes e Estimulantes) e ao CNPq pelas bolsas de iniciação científica.

\section{Referências}

Autran, E. S., Neves, I. A., Da Silva, C. S. B., Santos, G. K. N., Da Câmara, C. A. G., \& Navarro, D. M. A. F. (2009). Chemical composition, oviposition deterrent and larvicidal activities against Aedes aegypti of essential oils from Piper marginatum Jacq. (Piperaceae). Bioresource technology, 100(7), 22842288. https://doi.org/10.1016/j.biortech.2008.10.055

Brasil, Ministério do Meio Ambiente. (2012). Conservação in situ, ex situ e on farm. Obtido em https://www.mma.gov.br/biodiversidade/conservacao-epromocao-do-uso-da-diversidade-genetica/agrobiodiversidade/conserva\%C3\%A7\%C3\%A3o-in-situ,-ex-situ-e-on-farm.

Costa, J. D., Santos, P. F. D., Brito, S. A., Rodrigues, F. F., Coutinho, H. D., Botelho, M. A., \& Lima, S. G. D. (2010). Composição química e toxicidade de óleos essenciais de espécies de Piper frente a larvas de Aedes aegypti L. (Diptera: Culicidae). Latin American Journal of Pharmacy, 29(3), 463-467. ISSN $0326-2383$

Clement, C. R., Rocha, S. F. R., Cole, D. M., \& Vivan, J. L. (2007). Conservação on farm. Recursos genéticos vegetais, 511-543. ISBN 978-85-87697-34-9.

D'Angelo, L. C. A., Xavier, H. S., Torres, L. M. B., Lapa, A. J., \& Souccar, C. (1997). Pharmacology of Piper marginatum Jacq. planta medicinal popular utilizada como analgésico, antiinflamatório e hemostático. Fitomedicina, 4(1), 33-40. https://doi.org/10.1016/S0944-7113(97)80025-6

De Feo, V. (1991). Uso di piante ad azione antiinfiammatoria nell'Alto Ucayali, Perú Orientale. Fitoterapia, 62(6), $481-494$.

De Araújo Júnior, J. X., de Oliveira Chaves, M. C., da-Cunha, E. V. L., \& Gray, A. I. (1999). Cepharanone B from Piper tuberculatum. Biochemical Systematics and Ecology, 27(3), 325-327. https://doi.org/10.1016/S0305-1978(98)00083-0

De Queiroz, A. C., Alves, H. D. S., Cavalcante-Silva, L. H. A., Dias, T. D. L. M. F., Santos, M. D. S., Melo, G. M. D. A., Campesatto, E. A., de Oliveira Chaves, C. \& Alexandre-Moreira, M. S. (2014). Antinociceptive and anti-inflammatory effects of flavonoids PMT1 and PMT2 isolated from Piper montealegreanum Yuncker (Piperaceae) in mice. Natural product research, 28(6), 403-406. https://doi.org/10.1080/14786419.2013.867444

De Oliveira Chaves, M. C., \& de Oliveira Santos, B. V. (2002). Constituents from Piper marginatum fruits. Fitoterapia, 73(6), 547-549. https://doi.org/10.1016/S0367-326X(02)00167-3

Dos Santos Sales, V., do Nascimento, E. P., Brito Monteiro, Á., Nogueira da Costa, M. H., de Araújo Delmondes, G., Carneiro Soares, T. R., Soares, T. R. C.; Tintino, S. R.; Sobreira, F. R.; Figuêiredo, D. N.; Rodrigues, C. K. S.; Costa, J. G. M.; Coutinho, H. D. M.; Felipe, C. F. B.; Menezes, I. R. \& Kerntopf, M. R. (2017). Modulación in vitro de la actividad antibiótica por el aceite esencial de frutos de Piper tuberculatum Jacq. Revista Cubana de Plantas Medicinales, 22(1), 1-10. ISSN 1028-4796.

Guimarães, E. F., \& Giordano, L. C. D. S. (2004). Piperaceae do Nordeste brasileiro I: estado do Ceará. Rodriguésia, 55(84), 21-46. https://doi.org/10.1590/2175-78602004558402

Judd, W. S., Campbell, C. S., Kellogg, E. A., Stevens, P. F., \& Donoghue, M. J. (2009). Sistemática Vegetal: Um Enfoque Filogenético. Artmed Editora. ISBN 978-0-87893-407-2

Lameira, O. A., Cordeiro, I. M. C. C., \& Pires, H. C. G. (2020). Avaliação dos Descritores Morfoagronômico e Morfoanatomia da Lâmina Foliar de Pilocarpus: Microphyllus Stapf ex Wardleworth-Rutaceae, Ananas Comosus Var. Erectifolius (LB Smith) Coppens \& F. Leal-Bromeliacea e Psychotria Ipecacuanha (Brot.) Stokes. Editora Appris. ISBN 978-65-5523-293-6.

Melo, A., Araújo, A. A., \& Alves, M. (2013). Flora of Usina São José, Igarassu, Pernambuco: Aristolochiaceae and Piperaceae. Rodriguésia, 64(3), 543-553. ISSN 2175-7860.

Munsell, A. H. (1919). A Color Notation, 1905; 1907; with new preface, 1913; 1916, Geo. H. Ellis Co., Boston, Mass.

Pascoli, I. C., Dos Anjos, M. M., Silva, A. A., Lorenzetti, F. B., Cortez, D. A. G., Mikcha, J. M. G., Nakamura, T. U., Nakamura, C. V. \& Abreu Filho, B. A. Piperaceae extracts for controlling Alicyclobacillus acidoterrestris growth in commercial orange juice. Industrial Crops and Products, 116, 224-230. https://doi.org/10.1016/j.indcrop.2018.02.073 
Pereira, A. C. C., Castro, G. L. S., Rodrigues, P. C., Silva, G. B., Oliveira, D. A., \& Souza, C. R. B. (2019). An endophytic Pseudomonas sp. of Piper tuberculatum promotes growth on Piper nigrum through increase of root biomass production. Physiological and Molecular Phatology, 108. https://doi.org/10.1016/j.pmpp.2019.101420

Pereira, L. A., dos Santos, D. C., Rodrigues, P. F. A., Andrade, E. H. A. \& Guimarães, E. F. (2020). Valor de uso, indicações terapêuticas e perfil farmacológico e etnofarmacológico de duas espécies do gênero Piper L. em uma comunidade quilombola na Amazônia Oriental Brasileira. Brazilian Journal of Development, 6(7), 52027-52039. http://doi.org/10.34117/bjdv6n7-739

Pereira A. S., Shitsuka, D. M., Parreira, F. J., \& Shitsuka, R. (2018). Metodologia da pesquisa científica. [free e-book]. Santa Maria/RS. Ed. UAB/NTE/UFSM. ISBN 978-85-8341-204-5. ISBN 978-85-8341-204-5.

Perigo, C. V., Torres, R. B., Bernacci, L. C., Guimarães, E. F., Haber, L. L., Facanali, R., Vieira, M. A. R., Quecini, V. \& Marques, M. O. M. (2016). The chemical composition and antibacterial activity of eleven Piper species from distinct rainforest areas in Southeastern Brazil. Industrial crops and products, 94 , 528-539. https://doi.org/10.1016/j.indcrop.2016.09.028

Pinto, D. S., Duarte, F. M., IV Costa, J., de Almeida Filho, G., S Alves, H., Celia de O Chaves, M., \& de Luna F Pessoa, H. (2012). Antibacterial and hemolytic activities from Piper montealegreanum Yuncker (Piperaceae). Anti-Infective Agents, 10(1), 1-5. http:/doi.org/10.2174/2211362611201010001

Rocha, R. V da, Nunes, L. E., Fernandes, A. F. C., Catão, R. M. R. \& Alves, H. S. (2020). Atividade antimicrobiana e hemolítica de produtos obtidos de Piper montealegreanum Yuncker e efeito in vitro de Staphylococcus aureus. Research, Society and Development, 9(9), 1-19. https://doi.org/10.33448/rsd-v9i9.7410

Rodrigues, R. S., Hirt, A. P. M., \& Flores, A. S. (2012). Morfologia de plântulas das espécies de Rhynchosia (Leguminosae, Papilionoideae) de Roraima, Brasil. Acta Botanica Brasilica, 26(3): 585-592. https://doi.org/10.1590/S0102-33062012000300008.

Sarnaglia Junior, V. B., Bermudez, G. M. M., \& Guimarães, E. F. (2014). Diversity of Piperaceae at an Atlantic Forest remnant in the mountainous region of Espírito Santo, Brazil. Biotemas, 27(1), 49-57. https://doi.org/10.1590/S0102-33062012000300008.

Silva, D. F., Garcia, P. H. M., Santos, G. C. L., Farias, I. M. S. C., Pádua, G. V. G., Pereira, P. H. B., Da Silva, F. E., Batista, R. F., Neto, S. G. \& Cabral, A. M. D. (2021). Características morfológicas, melhoramento genético e densidade de plantio das culturas do sorgo e do milho: uma revisão. Research, Society and Development, 10(3), 1-9. https://doi.org/10.33448/rsd-v10i3.13172

Silva, D. D., Pinto, M. S. C., Gomes, R. N., Freitas, A., J. F., Aguiar, F. S., \& Pinto, M. G. C. (2020). Características morfológicas de frutos, sementes e plântulas de Luetzelburgia auriculata (Allemão) Ducke - Fabaceae. Research, Society and Development, 9(8), 1-19. https://doi.org/10.33448/rsd-v9i8.6133

Smith, J. F., Stevens, A. C., Tepe, E. J., \& Davidson, C. (2008). Placing the origin of two species-rich genera in the late cretaceous with later species divergence in the tertiary: a phylogenetic, biogeographic and molecular dating analysis of Piper and Peperomia (Piperaceae). Plant Systematics and Evolution, 275(1), 9-30. http://dx.doi.org/10.1007/s00606-008-0056-5

Sobreira, P. H. M., Hernandez, A. E. F., \& Souza, A. D. (2017). Inventário das espécies de piperaceae ocorrentes em três áreas de Porto Velho-RO e caracterização dos óleos essenciais de Piper tuberculatum jacq. com potencial ação de controle de fitopatógenos. Saber Científico, 6(1), 20-26. http://dx.doi.org/10.22614/resc-v6-n1-634

Vieira, E. A., Fialho, J. D. F., Silva, M. S., \& Faleiro, F. G. (2007). Variabilidade genética do banco ativo de germoplasma de mandioca do Cerrado acessada por meio de descritores morfológicos. Embrapa Cerrados-Boletim de Pesquisa e Desenvolvimento (INFOTECA-E). ISSN 1676-918X.

Yuncker, T. G. (1958). The Piperaceae - A family profile. Brittonia, 1-7. https://doi.org/10.2307/2804687

Yuncker, T. G. (1966). New species of Piperaceae from Brazil (No. 3). Instituto de Botânica.

Yuncker, T. G. (1972). The Piperaceae of Brazil. Hoehnea, 2, 19-366. 